\title{
Analysis of the Spatial Distribution of Identical and Two Distinct Virus Populations Differently Labeled with Cyan and Yellow Fluorescent Proteins in Coinfected Plants
}

\author{
Tsubasa Takahashi, Tomohiko Sugawara, Tsubasa Yamatsuta, Masamichi Isogai, \\ Tomohide Natsuaki, and Nobuyuki Yoshikawa
}

First and sixth authors: The 21st Century Center of Excellence Program, Iwate University, Morioka, 020-8550, Japan; second, third, fourth, and sixth authors: Faculty of Agriculture, Iwate University, Morioka, 020-8550, Japan; and fifth author: Faculty of Agriculture, Utsunomiya University, Utsunomiya, 321-8505, Japan.

Accepted for publication 23 April 2007.

\begin{abstract}
Takahashi, T., Sugawara, T., Yamatsuta, T., Isogai, M., Natsuaki, T., and Yoshikawa, N. 2007. Analysis of the spatial distribution of identical and two distinct virus populations differently labeled with cyan and yellow fluorescent proteins in coinfected plants. Phytopathology 97:1200-1206.

Apple latent spherical virus (ALSV) expressing yellow and cyan fluorescent proteins (ALSV-YFP and ALSV-CFP) was used to investigate the distribution of identical virus populations in coinfected plants. In Chenopodium quinoa plants inoculated with a mixture of ALSV-YFP and ALSV-CFP, fluorescence from YFP and CFP was always distributed separately in both inoculated and upper uninoculated leaves. Inoculation of each ALSV-YFP and ALSV-CFP to different leaves of a C. quinoa

plant resulted in the separate distribution of each virus population among different upper leaves. When $C$. quinoa leaves were first inoculated with ALSV-CFP and then ALSV-YFP was reinoculated into the same leaves at various times after the first inoculation, ALSV-YFP infected only tissues where ALSV-CFP infection had not been established. The spatial separation was also found in Nicotiana benthamiana leaves coinoculated with Bean yellow mosaic virus (BYMV)-YFP and BYMV-CFP. In contrast, both YFP and CFP fluorescence signals were observed in the same tissues of $N$. benthamiana leaves mixed infected with ALSV-YFP and BYMVCFP. YFP fluorescence from ALSV-YFP in mixed-infected leaves was brighter and longer than in leaves infected with ALSV-YFP singly.
\end{abstract}

In nature, mixed infection of different plant viruses is a common phenomenon in plants, especially in perennial plants like fruit trees. When two or more different viruses have infected the same plants, the viruses may replicate independently, or interact with each other either antagonistically or synergistically, depending on their combinations (6). In addition to mixed infections of different viruses, the virus genome that exists in a single-virusinfected plant is known to be heterogeneous, i.e., a mixture of sequence variants. For example, it is reported that all isolates of Apple stem grooving virus from several fruit trees contain at least two to four sequence variants that differ considerably from each other in nucleotide sequence $(12,13,28)$. Similar genome heterogeneity of an infecting virus in a single plant was reported in Apple chlorotic leaf spot virus and Apple stem pitting virus, and it may be a universal phenomenon in fruit tree viruses $(3,26)$. At present, there is no information on the distribution of sequence variants in infected leaves, e.g., on whether each sequence variant could replicate in the same cells and tissues or could be distributed separately in the different cells and tissues. The molecular maker of green fluorescent protein (GFP) from jellyfish, Aequorea victoria, a 27-kDa monomer protein (17), has been widely used for monitoring gene expression and protein localization because it is nontoxic to plants, brightly fluorescent, very stable in cells, and nondestructive (15). GFP has been used to investigate the localization of plant viruses in infected plants because the cell-to-cell and long-distance movement of viruses

Corresponding author: N. Yoshikawa; E-mail address: yoshikawa@iwate-u.ac.jp

doi:10.1094/PHYTO-97-10-1200

(c) 2007 The American Phytopathological Society can be observed directly in living cells and tissues by using GFPtagged plant viruses $(2,4,14,15,19,25)$. Furthermore, two different fluorescent proteins can be used for double-tagging techniques to analyze the interaction between two virus strains or two different viruses coinfecting the same plants. It has been recently reported that populations of identical, but differently labeled potyviruses, exhibited spatial separation in mixed-infected plants (5).

Apple latent spherical virus (ALSV) has an isometric virus particle of $25 \mathrm{~nm}$ in diameter and is classified into a new genus Cheravirus (9). ALSV has been isolated from apple trees in Japan without causing any obvious disease (11). The virus contains two single-stranded RNA species (RNA1 and RNA2) and three capsid proteins (Vp25, Vp20, and Vp24) (9,11). Recently, infectious cDNA clones of ALSV-RNAs were constructed and modified into viral vectors for expressing foreign genes in plants (10). The ALSV vector that expresses GFP has been used for demonstrating that a movement protein and the three capsid proteins are all indispensable for the cell-to-cell movement of the virus (27).

In this study, ALSV vectors expressing cyan fluorescent protein (CFP) or yellow fluorescent protein (YFP) were constructed and used to investigate the distribution of identical virus populations in coinfected plants. We also used Bean yellow mosaic virus (BYMV) expressing CFP or YFP to investigate the distribution of different virus populations in mixed-infected plants. The results indicated that identical but differently labeled virus populations (ALSV-YFP and ALSV-CFP or BYMV-YFP and BYMV-CFP) were always distributed separately in coinfected leaves. On the other hand, infection by different virus combinations (ALSV-YFP and BYMV-CFP) showed that both viruses were distributed in the same tissues of infected leaves. Furthermore, coinfection of ALSV and BYMV resulted in the synergistic effects consisting of severe symptoms and high ALSV accumulation. 


\section{MATERIALS AND METHODS}

Construction of the ALSV-RNA2 vectors expressing YFP and CFP. ALSV expressing YFP (ALSV-YFP) or CFP (ALSVCFP) was constructed from ALSV vectors (pEALSR1 and pEALSR2L5R5) (10). cDNA fragments containing YFP and CFP genes were amplified from pEYFP-1 and pECFP-1 (CLONTECH, Japan), respectively, by polymerase chain reaction (PCR) using EX Taq DNA polymerase (TAKARA, Japan) and primer pairs XhoGFP (5'-CCCTCGAGATGGTGAGCAAGGGCGAGGA-3', containing a XhoI site [underlined]) and BamGFP (5'-CGGGATCCCTTGTACAGCTCGTCCA-3', containing a BamHI site [underlined]), respectively (10). The amplified cDNA fragments of YFP and CFP genes were double-digested with XhoI and BamHI, and ligated to pEALSR2L5R5 restricted with the same enzymes. The resulting pEALSR2-based vectors containing YFP or CFP genes were designated pEALSR2L5R5YFP or pEALSR2L5R5CFP, respectively (Fig. 1A).

Construction of the BYMV-based vectors expressing YFP and CFP. CFP and YFP gene fragments were produced by PCR using two primers, Xba-EGFP (5'-CTAGTCTAGAATGGTGAGCAAGGGCGAGGAGCTG-3', containing a XbaI site [underlined]) and EGFP-Nhe (5'-CTAGCTAGCCTTGTACAGCTCGTCCATGCCGAGAG-3', containing a Nhe I site [underlined]) as described before. Each of the amplified products was digested with $X b a \mathrm{I}$ and NheI sites of plbG:Vec (23). The resulting pIbG:Vec containing CFP or YFP genes was designated pIbG:CFP and pIbG:YFP, respectively (Fig. 1B).

Viral inoculation. Both pEALSR2L5R5YFP and pEALSR2L5R5CFP were purified from large-scale cultures of Escherichia coli DH5a using a Qiagen plasmid mini kit (Qiagen, Japan). Each plasmid was mixed with purified pEALSR1 at a concentration of $1 \mu \mathrm{g} / \mu \mathrm{l}$ each, and mechanically inoculated to leaves of Chenopodium quinoa (6 true leaf stage). After a week, upper leaves $(0.1 \mathrm{~g})$ with symptoms were homogenized in $0.4 \mathrm{ml}$ of inoculation buffer $(0.1 \mathrm{M}$ Tris- $\mathrm{HCl}[\mathrm{pH} 7.8], 0.1 \mathrm{M} \mathrm{NaCl}$, and $\left.5 \mathrm{mM} \mathrm{MgCl} 2-6 \mathrm{H}_{2} \mathrm{O}\right)$ and then mechanically inoculated to host plant leaves.

In the case of BYMV-based vectors, pIbG:FL, pIbG-YFP, and pIbG-CFP were inoculated to Nicotiana benthamiana by a PDS1000/He Particle Delivery System (Bio-Rad, Hercules, CA) as described previously $(21,22)$. After a week, upper leaves $(0.1 \mathrm{~g})$ with mild mosaic symptoms were homogenized in $0.4 \mathrm{ml}$ of $0.1 \mathrm{M}$ phosphate buffer ( $\mathrm{pH}$ 7.0) and then mechanically inoculated to $N$. benthamiana leaves.

Fluorescence microscopy. Infected leaves of $C$. quinoa and $N$. benthamiana plants were observed under a fluorescent stereoscopic microscope (KEYENCE VB-G25, Japan) with the following filter sets: CFP-B filter sets (excitation filter $436 / 20 \mathrm{~nm}$, and barrier filter $480 / 30 \mathrm{~nm}$ ), and YFP-B filter sets (excitation filter $490 / 20 \mathrm{~nm}$, and barrier filter 540/40 nm). Digital micrographs were taken with a digital camera (Olympus DP70, Olympus, Japan). The images acquired with CFP and YFP filters were superimposed using Photoshop (Adobe).

ELISA. Virus accumulations in inoculated leaves were assessed by an enzyme-linked immunosorbent assay (ELISA). Infected samples were homogenized with carbonate-bicarbonate buffer, $\mathrm{pH} 9.6$, and trapped for $1 \mathrm{~h}$ at $37^{\circ} \mathrm{C}$ in ELISA plates. After incubation, the antibodies against ALSV or BYMV coat proteins diluted to $1 / 2,000$ in Tris-buffered saline containing Tween 20 (TTBS) were added and incubated overnight at $4^{\circ} \mathrm{C}$. The goat anti-rabbit immunoglobulin $\mathrm{G}$, diluted to $1 / 2,000$ in TTBS, was added and incubated for $1 \mathrm{~h}$ at $37^{\circ} \mathrm{C}$. Finally, $p$-nitrophenylphosphate $(1 \mathrm{mg} / \mathrm{ml})$ in $10 \%$ diethanolamine, $\mathrm{pH} 9.6$, was added, and the absorbance was measured with a microplate reader model 550 (Bio-Rad).

\section{RESULTS}

Distribution of identical but differently labeled virus populations in coinoculated plants. The mixtures of ALSV-CFP and ALSV-YFP were mechanically inoculated to leaves of $C$. quinoa plants, and the inoculated leaves were observed under a fluorescent microscope with the filters for CFP or YFP. Both CFP and YFP fluorescent spots first appeared on inoculated leaves at 3 days postinoculation (dpi) (Fig. 2A-(i)). Fluorescence spots were then enlarged as ring-shaped ones after 5 dpi (Fig. 2A-(ii) and (iii)) consistent with those of ALSV-GFP infection (27). When CFP images were superimposed on YFP images, fluorescence spots of CFP and YFP were always separated and never overlapped each other in coinoculated leaves, even if fluorescence spread over the whole area of leaves (Fig. 2A-(iii)). In upper uninoculated leaves, CFP and YFP fluorescence was initially found on veins and then spread over the whole area of leaf tissues.

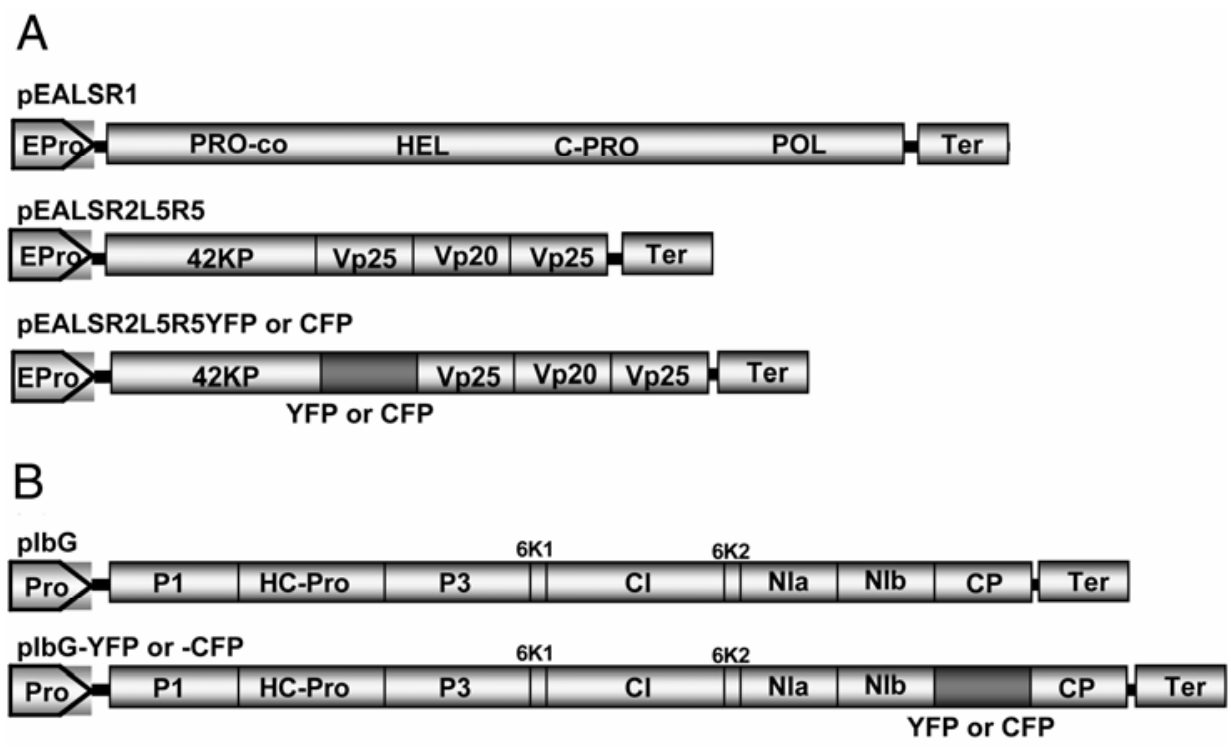

Fig. 1. Schematic representation of full-length infectious cDNA clones of the A, Apple latent spherical virus (ALSV) and B, Bean yellow mosaic virus (BYMV)based vectors. Cyan fluorescent protein (CFP) or yellow fluorescent protein (YFP) genes were inserted between 42KP and Vp25 genes of the ALSV vector, and between nuclear inclusion b (NIb) and coat protein (CP) genes of BYMV vector. EPro, enhanced CaMV 35S promoter; Pro, CaMV $35 \mathrm{~S}$ promoter; Ter, nopaline synthase terminator. 


\section{A}
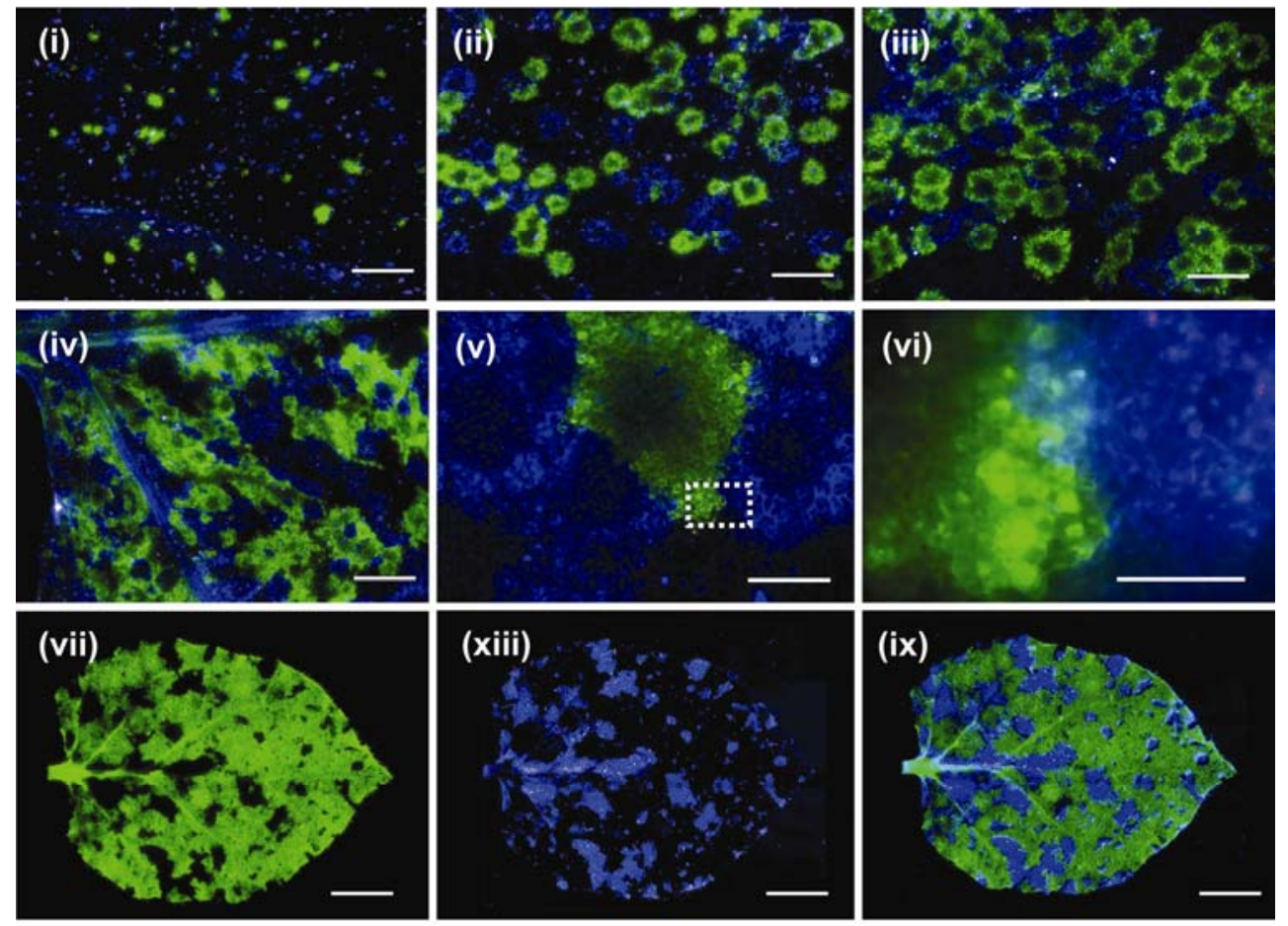

B
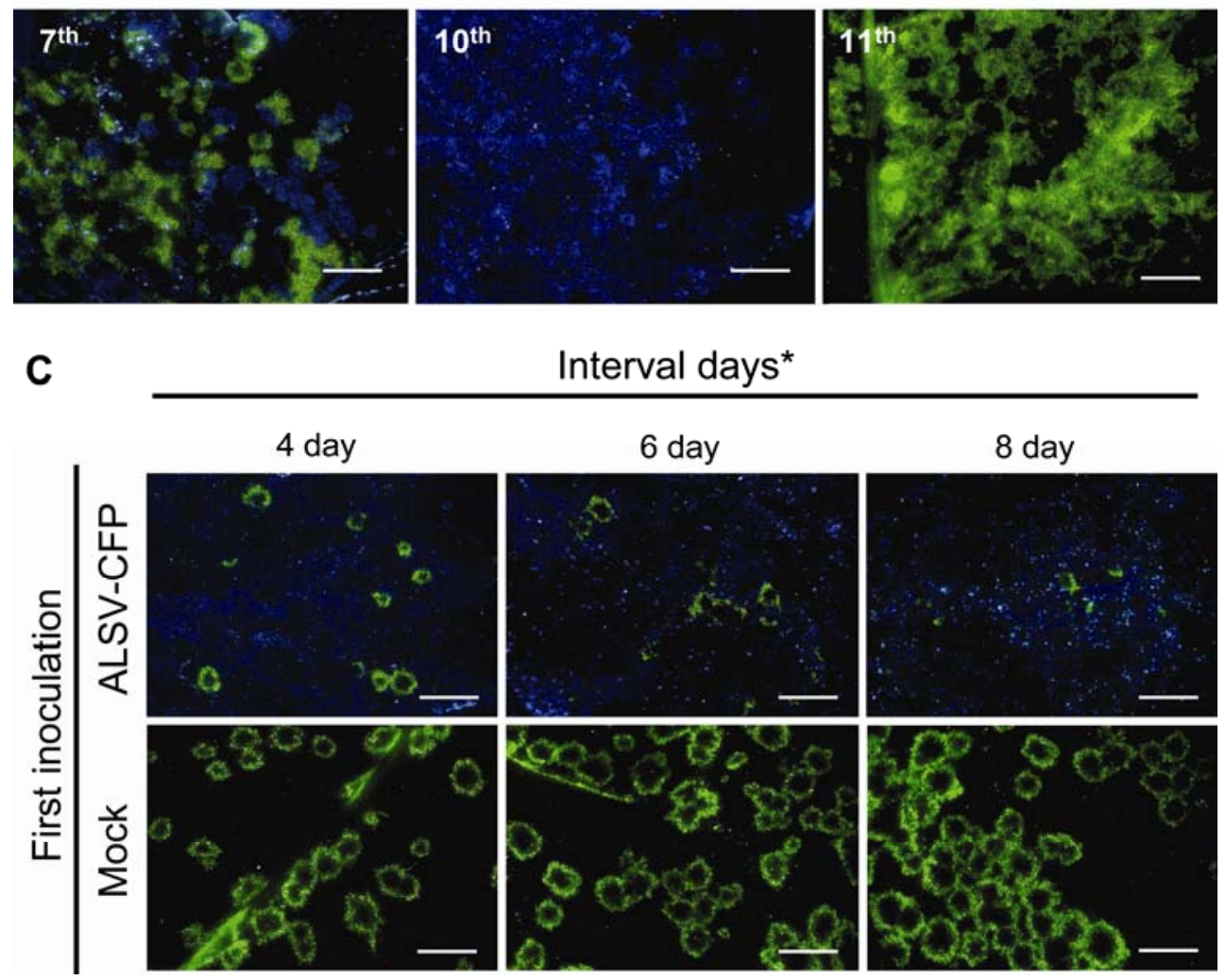

Fig. 2. Analysis of the distribution of identical, but differently labeled virus populations in coinoculated plants. A, The fluorescence of cyan fluorescent protein (CFP) or yellow fluorescent protein (YFP) on inoculated leaves at 3 days postinoculation (dpi) (i), 5 dpi (ii), 6 dpi (iii), and upper uninoculated leaves at 11 dpi (iv, v, and vi) of Chenopodium quinoa plants infected with Apple latent spherical virus expressing CFP (ALSV-CFP) and YFP (ALSV-YFP). A border area between populations of ALSV-YFP and ALSV-CFP in (v) is shown in (vi). The fluorescence of YFP (vii) and CFP (viii) on an upper leaf of Nicotiana benthamiana mixedinfected with Bean yellow mosaic virus expressing CFP (BYMV-CFP) and BYMV-YFP. The picture (ix) is a merged image of (vii) and (viii). B, Distributions of ALSV-CFP and ALSV-YFP among different upper leaves of a C. quinoa plant at 10 dpi. A C. quinoa plant was inoculated with ALSV-CFP to the 3rd and 5th true leaves and ALSV-YFP to the 4th and 6th leaves. Both YFP and CFP fluorescence signals were detected in a 7th leaf (left panel). CFP and YFP were separately distributed on the 10th (center panel) and on the 11th leaves (right panel), respectively. C, YFP spots at 7 days after ALSV-YFP inoculation on C. quinoa leaves that were first inoculated with ALSV-CFP or buffer only (Mock), and then secondly inoculated with ALSV-YFP at 4, 6, or 8 days after first inoculation. Bars: A, $2 \mathrm{~mm}$ in (i) to (iv), $500 \mu \mathrm{m}$ in (v), $200 \mu \mathrm{m}$ in (vi), and $5 \mathrm{~mm}$ in (vii) to (ix); $\mathbf{B}$ and $\mathbf{C}, 2 \mathrm{~mm}$. 
Fluorescence signals of CFP and YFP in upper leaves were also distributed separately and never overlapped each other (Fig. 2A(iv)). Observation of the adjacent regions between CFP and YFP fluorescence showed that only one or two cell layers of the border might have contained both CFP and YFP fluorescence (Fig. 2A(v) and (vi)). When a mixture of BYMV-CFP and BYMV-YFP was inoculated to $N$. benthamiana plant, spatial separation of virus populations was also found in upper leaves (Fig. 2A-(vii), (viii), and (ix)). These results indicated that populations of identical, but differently labeled viruses (ALSV-CFP and ALSVYFP or BYMV-CFP and BYMV-YFP), replicated predominantly in discrete areas and remained distributed separately. Our data

TABLE 1. Interference between Apple latent spherical virus expressing cyan fluorescent proteins (ALSV-CFP) and ALSV expressing yellow fluorescent proteins (YFP) on Chenopodium quinoa leaves which were first inoculated with ALSV-CFP and then with ALSV-YFP at different intervals

\begin{tabular}{|c|c|c|c|c|c|}
\hline \multirow{3}{*}{$\begin{array}{l}\text { Interval days between first } \\
\text { and second inoculation }\end{array}$} & \multirow{3}{*}{$\begin{array}{l}\text { Source of first } \\
\text { inoculation }\end{array}$} & \multicolumn{4}{|c|}{ Numbers of YFP spots ${ }^{\mathrm{a}}$} \\
\hline & & \multicolumn{4}{|c|}{ Leaf positions } \\
\hline & & 4th & 5 th & 6 th & 7 th \\
\hline \multirow[t]{2}{*}{4} & Mock & 135 & 193 & 1,002 & 511 \\
\hline & ALSV-CFP & 40 & 58 & 89 & 112 \\
\hline \multirow[t]{2}{*}{6} & Mock & 445 & 496 & 545 & 1,202 \\
\hline & ALSV-CFP & 130 & 169 & 166 & 137 \\
\hline \multirow[t]{2}{*}{8} & Mock & 262 & 366 & 750 & 1,447 \\
\hline & ALSV-CFP & 41 & 41 & 12 & 0 \\
\hline
\end{tabular}

a The sum of YFP spots per two leaves counted at 7 days after ALSV-YFP inoculation.

A
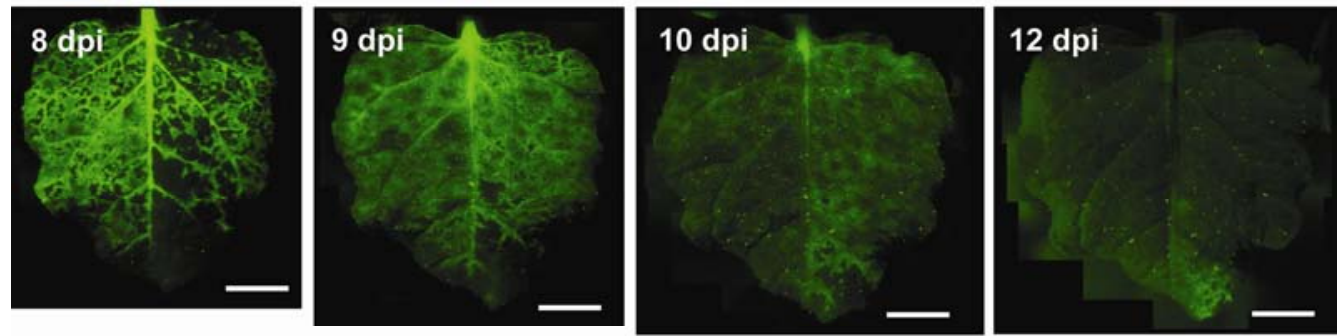

B
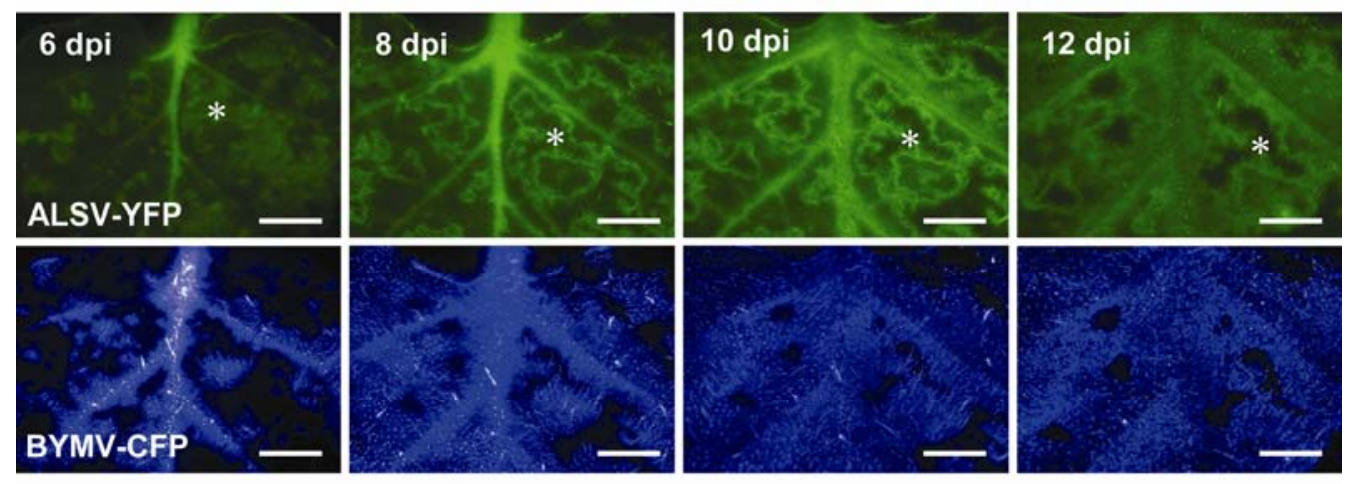

C
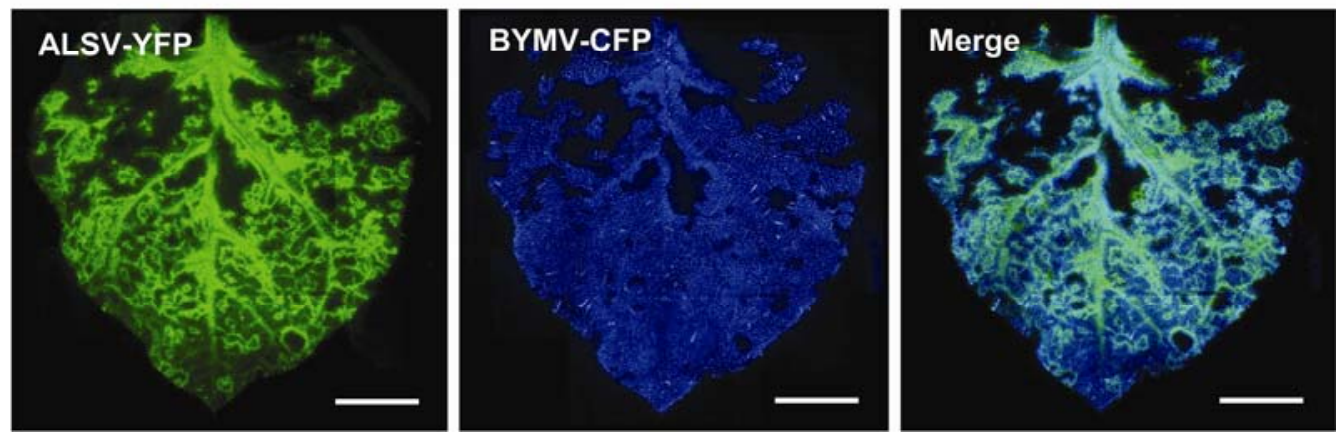

Fig. 3. Distribution of Apple latent spherical virus expressing yellow fluorescent protein (ALSV-YFP) and Bean yellow mosaic virus expressing cyan fluorescent protein (BYMV-CFP) in coinfected plants. A, YFP fluorescence on the same inoculated leaf of an Nicotiana benthamiana plant infected with ALSV-YFP at 8, 9, 10, and 12 days postinoculation (dpi). B, Distribution of YFP and CFP of an upper leaf of an N. benthamiana plant coinfected with ALSV-YFP and BYMV-CFP. The same uninoculated upper leaf was photographed at 6, 8, 10, and 12 dpi. Asterisks indicate the same position. C, Distribution of YFP and CFP in an upper leaf of $N$. benthamiana coinfected with ALSV-YFP and BYMV-CFP at 12 dpi. Bars: A and C, $5 \mathrm{~mm}$, and $\mathbf{B}, 2 \mathrm{~mm}$. 
were consistent with the spatial separation of three different potyviruses and a Potato virus $X$ (PVX) population in mixed-infected $N$. benthamiana plants (5).

Separate distribution of ALSV-CFP and ALSV-YFP among different upper leaves. In the next experiments, ALSV-CFP and ALSV-YFP were separately inoculated to the 3rd and 5th true leaves and to the 4 th and 6th true leaves of $C$. quinoa plants (7 true leaf stage), respectively. Observation by fluorescent microscope showed that both CFP and YFP fluorescence signals were found in a separated area in a 7th true leaf at 10 dpi (Fig. 2B). However, in the 8th to 15 th true leaves after $10 \mathrm{dpi}$, each CFP and YFP fluorescence was observed in different leaves, i.e., ALSVCFP was distributed in the 8th, 10th, 12th, and 14th leaves, and ALSV-YFP was detected in the 9th, 11th, 13th, and 15th leaves (Fig. 2B). The experiment was repeated two times and the results were essentially identical. These results clearly indicated that the spatial separation of ALSV-CFP and ALSV-YFP occurred among different leaves of infected $C$. quinoa plants.

Interference between ALSV-CFP and ALSV-YFP. To investigate the interference between ALSV-CFP and ALSV-YFP in infected leaves, ALSV-CFP was first inoculated to the 4th to 7 th true leaves of $C$. quinoa plants ( 8 true leaf stage), and secondly ALSV-YFP was inoculated to the same leaves at 4, 6, and 8 days after first inoculation. Other sets of $C$. quinoa plants were inoculated with buffer only (mock) followed by a second inoculation with ALSV-YFP. The development and distribution of each virus population in inoculated leaves were observed at 7 days after a second inoculation by fluorescent microscope. The YFP fluorescence with roughly circular dimensions (YFP spots) was observed in all cases of mock-inoculated leaves (Fig. 2C; Table 1). The sizes of YFP spots in mock-inoculated leaves were almost identical irrespective of the interval days between first and second inoculation (Fig. 2C). In contrast, YFP spots on leaves preinoculated with ALSV-CFP were always fewer in number and smaller in size than those on mock-inoculated leaves, and these YFP spots were only found in tissue areas where CFP fluorescence was not observed (Fig. 2C). When the interval between the first and second inoculations was $8 \mathrm{dpi}$, ALSV-YFP infection was extremely reduced in the 6th and 7th leaves compared with those in mock-inoculated leaves (Table 1). The results indicated that preinfection of ALSV-CFP interferes with effective infection of ALSVYFP. In other words, ALSV-YFP could not infect the tissue area where ALSV-CFP infection had already been established.

Distribution of different virus populations in coinfected plants. When $N$. benthamiana plants were inoculated with ALSVYFP, the YFP fluorescence was observed initially on veins in upper leaves at $5 \mathrm{dpi}$ and then found on the whole tissues of upper leaves (Fig. 3A). However, the intensity of YFP fluorescence was so weak compared with that in infected C. quinoa plants, and the fluorescence rapidly disappeared after 10 dpi (Fig. 3A). When ALSV-YFP and BYMV-CFP were mechanically inoculated to each half leaf of $N$. benthamiana, only a few YFP and CFP spots were found in inoculated leaves at $3 \mathrm{dpi}$, and the intensity of fluorescence was too weak to analyze their distribution in inoculated leaves. In upper leaves, CFP and YFP fluorescence signals were detected initially on veins at 5 dpi (data not shown) and then spread to other area of leaves at 6 to $12 \mathrm{dpi}$ (Fig. 3B). The YFP fluorescence in mixed-infected leaves was always brighter and maintained longer than in leaves infected with ALSV-YFP singly. In Figure 3, the exposure time for YFP fluorescence in a leaf infected with ALSV-YFP singly (Fig. 3A) was four times longer than those in a leaf mixed infected with ALSV-YFP and BYMVCFP (Fig. 3B). The distribution pattern of CFP fluorescence almost coincided with that of YFP fluorescence, strongly suggesting that ALSV-YFP and BYMV-CFP could exist in the same tissues in upper leaves of $N$. benthamiana plants (Fig. 3C).

Synergistic interaction between ALSV and BYMV. The accumulation levels of ALSV and BYMV in the inoculated and uninoculated upper leaves of $N$. benthamiana plants infected with a single virus or two viruses were examined by ELISA at $14 \mathrm{dpi}$. ALSV accumulation levels in leaves doubly infected with ALSV and BYMV were two to threefold higher than those in leaves infected with ALSV singly (Fig. 4). On the other hand, there were no differences in BYMV accumulation levels between BYMVinfected leaves and the two virus-infected (ALSV and BYMV) leaves (Fig. 4).

To investigate why ALSV accumulation levels were higher in mixed-infected plants than those in single-infected plants, the helper component proteinase (HC-Pro) sequence of BYMV was ligated to the region between the $42 \mathrm{~K}$ and Vp25-encoding sequences of the ALSV-RNA2 vector genome (Fig. 1A), and the resulting ALSV-BYHCPro was inoculated to $C$. quinoa plants (8 true leaf stage) by mechanical inoculation. Infected $C$. quinoa plants showed more severe mosaic symptoms in the upper leaves at 12 to 14 dpi (data not shown) compared with those in plants infected with ALSV. ELISA of inoculated leaves at 14 dpi indicated that the accumulation level of ALSV-BYHCPro was two to three times higher than that of ALSV (data not shown), strongly suggesting that the HC-Pro of BYMV might be a factor for enhancement of ALSV accumulation levels in mixed-infected plants.

\section{DISCUSSION}

The results presented in this paper showed that populations of identical, but differently labeled viruses (ALSV-CFP and ALSVYFP or BYMV-CFP and BYMV-YFP), replicated predominantly
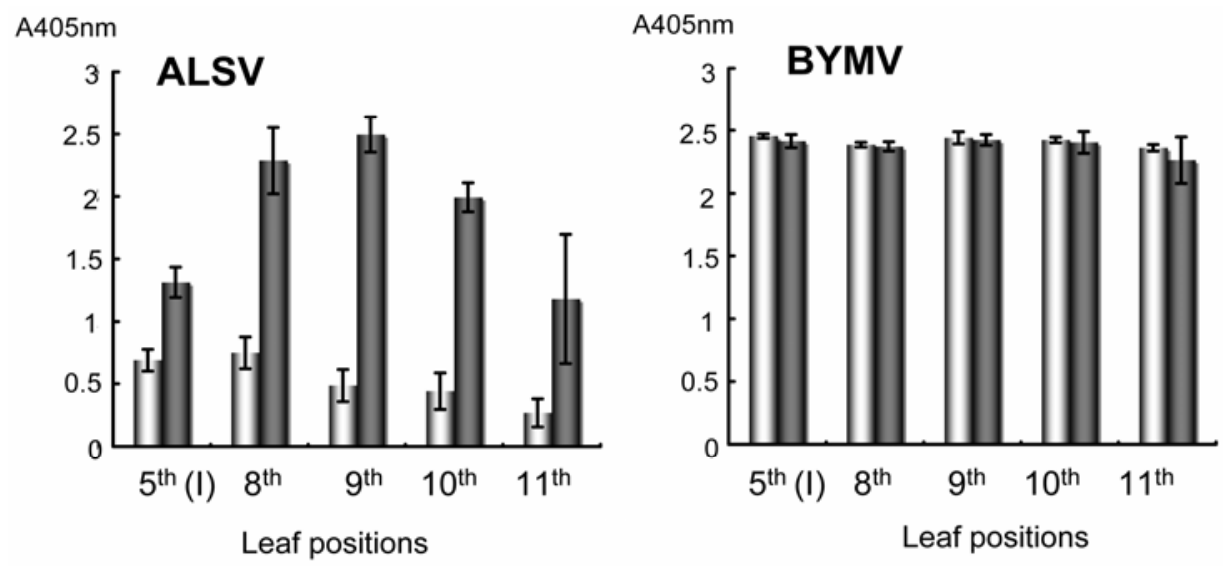

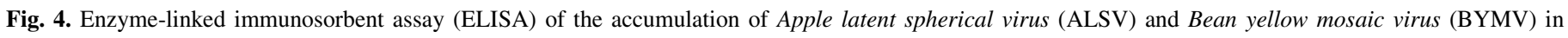

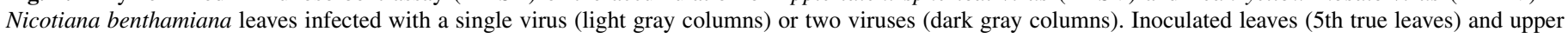
uninoculated leaves (8th to 11 th true leaves) were assayed at 14 days postinoculation. 
in discrete areas and remained separately distributed in both inoculated and upper uninoculated leaves in coinoculated plants. This was consistent with the spatial separation of three different potyviruses and PVX using viruses differently labeled with GFP and DsRed (5). The separated distributions of identical viruses in coinfected leaves were also analyzed by electron microscopy for Alfalfa mosaic virus and in situ hybridization for Cucumber mosaic virus $(7,24)$. Thus, the spatial separation between populations of identical viruses and strains in infected plants might be a common phenomenon, though the mechanism(s) of the spatial separation is still unknown.

In plants infected with ALSV-YFP and ALSV-CFP, both YFP and CFP fluorescence signals were observed in a few border cells between two different fluorescent cell clusters (Fig. 2A-(v) and (vi)). A similar observation was reported in infected tissues with populations of identical but different labeled potyviruses at border areas of two neighboring, different colored cell clusters (5). The border cells containing both YFP and CFP fluorescence signals might have been the cells in which ALSV-YFP and ALSV-CFP initiated infection to simultaneously, because a secondary virus could not infect the cells where a primary virus infection has already been established as discussed below.

In our studies, the spatial separation of ALSV-CFP and ALSVYFP also occurred among leaves of infected $C$. quinoa plants when ALSV-CFP and ALSV-YFP were separately inoculated to different leaves of plants (Fig. 2B). In other words, the spatial separation occurs not only at the cellular level in nonvascular tissues but also within the vascular tissue in infected $C$. quinoa plants. It is thought that ALSV-CFP replicated in the 3rd and 5th true leaves might have moved to upper leaves through phloem bundles differently from those utilized by ALSV-YFP in the 4th and 6th true leaves. When ALSV-CFP moved to the upper leaves faster than ALSV-YFP and established the infection in vascular tissues, the slower migrating virus (ALSV-YFP) might not have been able to infect the upper leaves.

When leaves of $C$. quinoa plants were first inoculated with ALSV-CFP, and then ALSV-YFP was secondly inoculated to the same leaves, ALSV-YFP infection was strictly restricted to the tissues where ALSV-CFP infection had not been established (Fig. 2C). The results indicated that a secondary virus (ALSV-YFP) could not infect the tissues where a primary virus (ALSV-CFP) infection had been established. The spatial separation and interference between ALSV-CFP and ALSV-YFP in this paper is thought to indicate visually the phenomenon of cross-protection (16).

In contrast, both YFP and CFP fluorescence signals were observed in the same area of $N$. benthamiana leaves mixed infected with ALSV-YFP and BYMV-CFP (Fig. 3B and C), strongly suggesting that both viruses were replicated in the same tissues of infected leaves. In addition, YFP fluorescence in leaves mixed infected with ALSV-YFP and BYMV-CFP was brighter than in leaves infected with ALSV-YFP singly, and it also remained longer than that in single infection. The enhancement of YFP fluorescence is thought to be due to the increase of ALSV-YFP accumulation in mixed-infected leaves, because the accumulation level of ALSV in leaves infected with ALSV and BYMV was two- to threefold higher than in leaves infected with ALSV singly (Fig 4). These results indicated that the synergistic interaction between ALSV and BYMV occurred in mixed-infected $N$. benthamiana plants as reported for other members of the genus Potyvirus $(18,20)$. The synergistic interaction between ALSV and BYMV reported here is thought to be caused by the suppression of posttranscriptional gene silencing with the HC-Pro encoded by the BYMV genome $(1,8)$. This was clearly demonstrated by the inoculation of $C$. quinoa plants with ALSV-BYHCPro containing a BYMV HC-Pro gene in which the accumulation level of ALSVBYHCPro was two- to threefold higher than that in leaves infected with ALSV (data not shown).

\section{ACKNOWLEDGMENTS}

This work was supported in part by the 21st Century Center of Excellence Program and a research grant (no. 15380032) from the Ministry of Culture, Sports, Science, and Technology of Japan.

\section{LITERATURE CITED}

1. Anandalakshmi, R., Pruss, G. J., Ge, X., Marathe, R., Mallory, A. C., Smith, T. H., and Vance, V. B. 1998. A viral suppressor of gene silencing in plants. Proc. Natl. Acad. Sci. USA 95:13079-13084.

2. Baulcombe, D. C., Chapman, S., and Cruz, S. S. 1995. Jellyfish green fluorescent protein as a reporter for virus infections. Plant J. 7:10451053.

3. Candresse, T., Lanneau, M., Revers, F., Macquaire, G., German, S., Grasseau, N., Dunez, J., and Malinowski, T. 1995. An immunocapture PCR assay adapted to the detection and the analysis of the molecular variability of the Apple chlorotic leaf spot virus. Acta Hortic. 386:136147.

4. Casper, S. J., and Holt, C. A. 1996. Expression of the green fluorescent protein-encoding gene from tobacco mosaic virus-based vector. Gene 173:69-73.

5. Dietrich, C., and Maiss, E. 2003. Fluorescent labeling reveals spatial separation of potyvirus populations in mixed infected Nicotiana benthamiana plants. J. Gen. Virol. 84:2871-2876.

6. Hull, R. 2002. Matthews' Plant Virology. 4th ed. Academic Press, San Diego.

7. Hull, R., and Plaskitt, A. 1970. Electron microscopy on the behaviour of two strains of alfalfa mosaic virus in mixed infections. Virology 42:773776.

8. Kasschau, K. D., and Karrington, J. C. 1998. A counterdefensive strategy of plant viruses: Suppression of posttranscriptional gene silencing. Cell 95:461-470.

9. Le Gall, O., Iwanami, T., Karasev, A. V., Jones, T., Lehto, K., Sanfacon, H., Wellink, J., Wetzel, T., and Yoshikawa, N. 2005. Genus cheravirus. In: VIIIth Report of the ICTV on Taxonomy of Viruses. Elsevier Academic Press, San Diego.

10. Li, C., Sasaki, N., Isogai, M., and Yoshikawa, N. 2004. Stable expression of foreign proteins in herbaceous and apple plants using Apple latent spherical virus RNA2 vectors. Arch. Virol. 149:1541-1558.

11. Li, C., Yoshikawa, N., Takahashi, T., Ito, T., Yoshida, K., and Koganezawa, H. 2000. Nucleotide sequence and genome organization of apple latent spherical virus: New virus classified into the family Comoviridae. J. Gen. Virol. 81:541-547.

12. Magome, H., Yoshikawa, N., and Takahashi, T. 1999. Single-strand conformation polymorphism analysis of apple stem grooving capillovirus sequence variants. Phytopathology 89:136-140.

13. Magome, H., Yoshikawa, N., Terauchi, H., and Takahashi, T. 1997. Analysis of the double stranded RNA in tissues infected with apple stem grooving capillovirus. Ann. Phytopathol. Soc. Jpn. 63:450-454.

14. Oparka, K. J., Roberts, A. G., Prior, D. A. M., Chapman, D., Baulcombe, D. S., and Santa Cruz, S. 1995. New ideas in cell biology. Protoplasma 189:133-141.

15. Oparka, K. J., Roberts, A. G., Santa Cruz, S., Boevink, P., Prior, D., and Smallcombe, A. 1997. Using GFP to study virus invasion and spread in plant tissues. Nature 388:401-402.

16. Pennazio, S., Roggero, P., and Conti, M. 2001. A history of plant virology. Cross protection. Microbiologica 24:99-114.

17. Prasher, D. C., Eckenrode, V. K., Ward, W. W., Prendergast, F. G., and Cormier, M. J. 1992. Primary structure of the Aquorea Victoria greenfluorescent protein. Gene 111:229-233.

18. Pruss, G., Ge, X., Shi, X. M., Carrington, J. C., and Vance, V. B. 1997. Plant viral synergism: The potyviral genome encodes a broad-range pathogenicity enhancer that transactivates replication of heterologous viruses. Plant Cell 9:859-868.

19. Roberts, A. G., Santa Cruz, S., Roberts, I. M., Prior, D. A. M., Turgeon, R., and Oparka, K. J. 1997. Phloem unloading in sink leaves of Nicotiana benthamiana: Comparison of a fluorescent solute with a fluorescent virus. Plant Cell 9:1381-1396.

20. Rochow, W. F., and Ross, A. F. 1955. Virus multiplication in plants doubly infected by potato viruses $\mathrm{X}$ and $\mathrm{Y}$. Virology 1:10-27.

21. Satoh, H., Matsuda, H., Kawamura, T., Isogai, M., Yoshikawa, N., and Takahashi, T. 2000. Intercellular distribution, cell-to-cell trafficking and tubule-inducing activity of the $50-\mathrm{kDa}$ movement protein of Apple chlorotic leaf spot virus fused to green fluorescent protein. J. Gen. Virol. 81:2085-2094.

22. Satoh, H., Yoshikawa, N., and Takahashi, T. 1999. Construction and biolistic inoculation of an infectious cDNA clone of apple chlorotic leaf spot trichovirus. Ann. Phytopathol. Soc. Jpn. 65:301-304. 
23. Takahashi, T., Nagaoka, E., Satoh, C., Kinoshita, S., Okuda, S., Kosaka, Y., and Natsuaki, T. 2004. Dynamic study of bean yellow mosaic virus carrying GFP in host plants. (Abstr.) Jpn. J. Phytopathol. 70:266.

24. Takeshita, M., Shigemune, N., Kikuhara, K., Furuya, N., and Takanami, Y. 2004. Spatial analysis for exclusive interactions between subgroups I and II of Cucumber mosaic virus in cowpea. Virology 328:45-51.

25. Wang, H. L., Sudarshana, M. R., Gilbertson, R. L., and Lucas, W. J. 1999. Analysis of cell to cell and long-distance movement of Bean dwarf mosaic geminivirus-green fluorescent protein reporter in host and nonhost species: Identification of site of resistance. Mol. Plant-Microbe Interact. 12:345-355.
26. Yoshikawa, N., Matsuda, H., Oda, Y., Isogai, M., and Takahashi, T. 2001. Genome heterogeneity of Apple stem pitting virus in apple. Acta Hortic. 550:285-290.

27. Yoshikawa, N., Okada, K., Asamuma, K., Watanabe, K., Igarasi, A., Li, C., and Isogai, M. 2006. A movement protein and three capsid proteins are all necessary for the cell-to-cell movement of apple latent spherical cheravirus. Arch. Virol. 151:837-848.

28. Yoshikawa, N., Sasamoto, K., Sakurada, M., Takahashi, T., and Yanase, H. 1996. Apple stem grooving and citrus tatter leaf capilloviruses obtained from a single shoot of Japanese pear (Pyrus serotina). Ann. Phytopathol. Soc. Jpn. 62:119-124. 